Article

\title{
Dynamic Duty-Cycle MAC Protocol for IoT Environments and Wireless Sensor Networks
}

\author{
Gayoung Kim ${ }^{1}$, Jin-Gu Kang ${ }^{1}$ (D) and Minjoong Rim ${ }^{2, *}$ \\ 1 Department of Computer Science and Engineering, Dongguk University, Seoul 04620, Korea; \\ dolga2000@dgu.edu (G.K.); kanggu12@dongguk.edu (J.-G.K.) \\ 2 Department of Information and Communication Engineering, Dongguk University, Seoul 04620, Korea \\ * Correspondence: minjoong@dgu.edu; Tel.: +82-2-2260-3595
}

Received: 31 August 2019; Accepted: 23 October 2019; Published: 25 October 2019

check for updates

\begin{abstract}
This paper proposes a new protocol that can be used to reduce transmission delay and energy consumption effectively. This will be done by adjusting the duty-cycle (DC) ratio of the receiver node and the contention window size of the sender node according to the traffic congestion for various devices in the Internet of Things (IoT). In the conventional duty-cycle MAC protocol, the data transmission delay latency and unnecessary energy consumption are caused by a high collision rate. This is because the receiver node cannot sufficiently process the data of the transmitting node during the traffic peak time when the transmission and reception have the same duty-cycle ratio. To solve this problem, this paper proposes an algorithm that changes the duty-cycle ratio of the receiver and broadcasts the contention window size of the senders through Early Acknowledgment (E-ACK) at peak time and off/peak time. The proposed algorithm, according to peak and off/peak time, can transmit data with fewer delays and minimizes energy consumption.
\end{abstract}

Keywords: green energy; asynchronous duty-cycle MAC protocol; low energy consumption; low transmission delay; Internet of Things

\section{Introduction}

Recently, due to the rapid increase in sensor devices, a large amount of reliable data transmission has required the use of limited energy. Thus, it is urgent to develop a protocol that efficiently processes data with a high amount of devices and energy in the Internet of Things (IoT) environment [1]. IoT devices are mostly occupied by sensor networks that require minimum energy consumption and low transfer delay. These devices should be able to transmit data as efficiently as possible. In wireless sensor networks (WSNs) [2], heavy traffic occurs, or data rapidly change, for a short period, and it is also frequently lost. Therefore, research efforts to reduce transmission delay by minimizing energy consumption for devices in WSNs or IoT environments. Typical techniques used in the research are duty-cycling [3].

Duty-cycling technology supports periodic switching to listen and sleep modes to reduce energy consumption when events occur for IoT devices. Listen mode consumes less power than other protocols that consume power for communication but do not consume energy in sleep mode. In a WSN, the duty-cycle MAC protocol is divided into a sleep interval and a listening interval, which can monitor and collect data with less energy when events occur. It is possible to detect events and collect data in various environments by sending information on electricity, water, or gas to users [4]. Reliable data collection and detection is crucial for data transmission with minimum energy and delay because data must be transmitted without delay in the event of fire in areas where it is difficult to replace batteries.

However, the periodic switching mode changes to the listen mode for communication even when there is no event. This results in an unnecessary wastage of energy. Data transmission in 
an environment with traffic congestion causes problems of transmission delay, and the entire service life of the application is shortened due to the collision. Thus, the use of the existing duty-cycle (DC) MAC protocol [5] is not suitable. Therefore, it is necessary to develop a protocol for effective event detection [6] and data collection [7] considering various traffic conditions. For example, if the total number of wake-ups to listen to an application is determined [8], the existing duty-cycle MAC occurs irrespective of the congestion occurring at the time of the traffic. This reduces data collisions and transmission delays if the receiver wakes up frequently only at times in heavy traffic.

The proposed algorithm increases the number of wake-up times by reducing the sleep time of the receiver at the peak traffic time and reduces the number of wake-up in the opposite case. This demonstrates reduced energy consumption. This paper proposes a new duty-cycle based MAC protocol suitable for congested WSNs and IoT. According to the degree of network traffic congestion, the duty-cycle ratio of the receiver and the contention window size information of the senders are broadcast through E-ACK (Early Acknowledgment), which reduces the transmission latency and waste of energy.

The composition of the paper is described in detail in Section 1, followed by a summary of related work in Section 2. Section 3 proposes the operation procedure of the MAC layer. In Section 4, mathematical modeling is performed to predict the proposed algorithm and performance. In Section 5 , we compare the performance of the proposed algorithm with the energy consumption and average service latency. Finally, Section 6 presents conclusions and future research topics.

\section{Environment of Duty-Cycled System}

There are various protocols for saving energy in IoT devices using the MAC protocol in sensor network environments. Using limited energy periodically, intermittent devices that transmit and monitor data have a short life because of high traffic for a certain period. To solve this problem, it is essential to develop a protocol that minimizes energy according to traffic congestion.

\subsection{Smart Meters}

Smart meters [9] are digital electronic devices that collect information about the use of electricity, water, or gas and that transmit it safely to utilities [10]. It can improve the network efficiency by monitoring contents in real time and estimating the future data transmission pattern through the data collected by the end user. Managing the consumption of data through the smart meter enables real-time monitoring of the impact on the network. However, since the battery life of the smart meter is limited, it is necessary to limit the amount and frequency of the transmission data.

\subsection{Duty-Cycle MAC Protocols}

Due to the increase in the economic efficiency of the wireless sensor network, it is necessary to use energy efficiently for a limited period of time. In the MAC layer protocol, the DC can reduce power consumption by repeating many sleep and wake-up methods. The synchronous MAC protocol [11] broadcasts a beacon in every cycle and shares the schedule information. The represented protocols are S-MAC [12], T-MAC [13], and D-MAC [14]. The synchronous approach allows for data transmission without long delays because all neighboring nodes wake up at the same time.

In order to use the channel efficiently, all nodes share each other's schedule information and transmit data at different times, so that data collision, idle listening, and overhearing can be eliminated or limited. However, even if the transmission is unnecessary, the coordinator broadcasts the beacon every cycle, which causes energy wastage due to unnecessary sync packet transmission and wake-up for synchronization.

Since the asynchronous MAC protocol [15] operates on its own schedule, it provides a simple operational method. The synchronous method can solve the problems caused by the large amount of data to be transmitted through the channel reservation. However, when there is little data to send or irregular periods, overhead occurs. In addition, since data is transmitted through channel reservation, 
it indicates a high level of consumption in terms of cost and energy use. However, asynchronous operation in an environment where events do not occur frequently can eliminate the overhead, which is a problem of synchronicity that occurs through the transmission of a preamble that uses little energy in order to wake up the nodes. Typical asynchronous MAC protocols are B-MAC [16], X-MAC [17], and Wise MAC [18].

The reason why they do not know each other's schedule is due to the disadvantage of data transmission to other nodes. This is because the channel is used until the transmission delay time of the maximum duty-cycle period per link and the E-ACK of the preamble are received. Therefore, this protocol is not suitable for environments where data must be transmitted within a short period of time. Figure 1 below compares the legacy X-MAC and S-MAC operation methods that are asynchronous and synchronous.

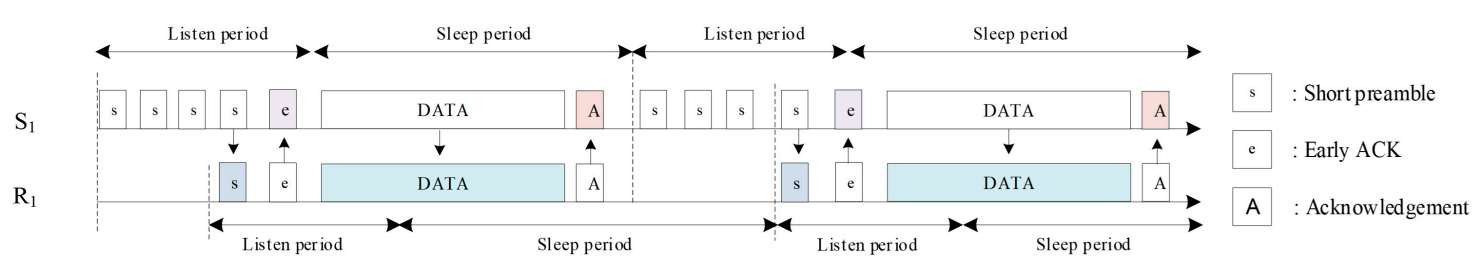

(a)

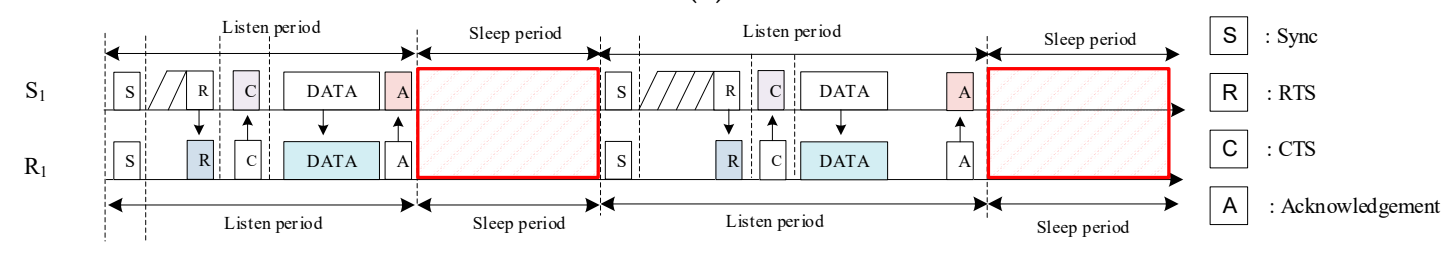

(b)

Figure 1. Duty-cycle-based protocol operation methods: (a) X-MAC protocol; (b) S-MAC protocol.

The asynchronous and synchronous duty-cycle MAC protocols increase the probability of data collision when many events occur in the network topology 1:N because the duty-cycle ratio of the transmitting and receiving nodes always work in the same way.

Due to retransmission, there are problems of energy loss and transmission delay in a heavy traffic environment, and this increases the data collision of several transmitting nodes by one receiving node. In order to support various IoT devices, WSNs require an advanced protocol that can improve energy and delay transmission according to traffic change.

\subsection{Monitoring Systems}

Industrial and environmental monitoring [19] requires an environment that can collect and process massive amounts of data in a wireless sensor network systems. There is need for a protocol on data transmission collected through the application of monitoring and managing the standby state and weather conditions. In the IoT environment, all the sensor nodes transmit the collected data from the sensor node to the sink node using the wireless Internet. Sink nodes are constantly supplied with energy, but other sensor nodes are necessarily supplied with a limited amount of energy, since they must consume minimal energy.

In the future, it is expected that protocols for monitoring systems using IoT devices will require the development of protocols that can transmit data in real time with minimum energy in various traffic environments.

\subsection{MAC Protocol for Wireless Body Area Networks}

Wireless body area networks (WBANs) [20] are low-cost, compact body devices that sense human body parameters (temperature, brain activity, and heart rate) [19] and deliver information gathered 
to end stations. Body nodes (BNs) constitute the WBAN: A BN is a wearable node that is inserted into the human body. It is required that the energy supply is limited and the information about body parameters is transmitted without delay. Most WBAN MAC protocols do not transfer energy efficiently due to the delay and packet drop in many situations. Additionally there is a time limit to determining the right decision because of the short time on unexpected traffic roads. For this reason, this study focuses on a MAC protocol that minimizes transmission delays and reduces unnecessary energy.

\section{The Proposed DDC-MAC Network Model}

\subsection{The System Model}

The dynamic duty-cycle MAC protocol (DDC-MAC) is a MAC protocol considering IoT environments. If the existing sensor network can communicate based on the number of $D_{1}$ sensor nodes, this paper considers an environment with a number of $D_{2}$ sensor nodes in the IoT environment of the DDC-MAC that can communicate with one coordinator. Figure 2a below shows that only $D_{1}$ devices transmit data during off/peak time, and $D_{2}$ devices try to transmit data when peak time is reached.

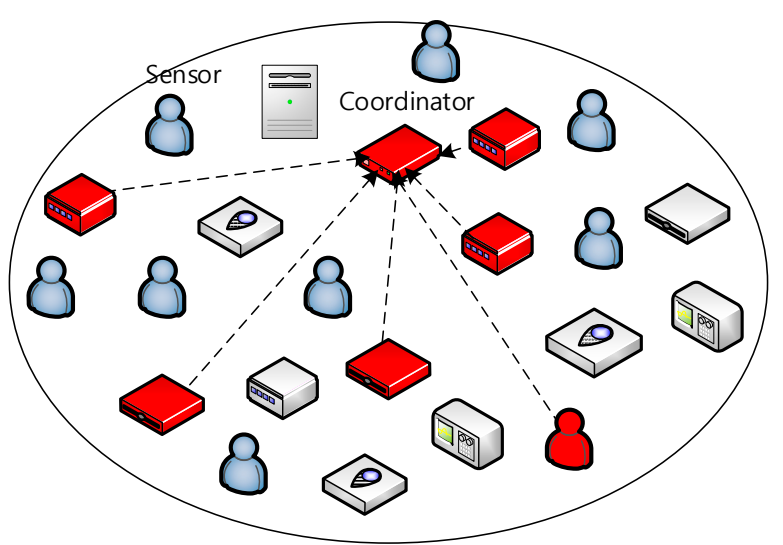

(a)

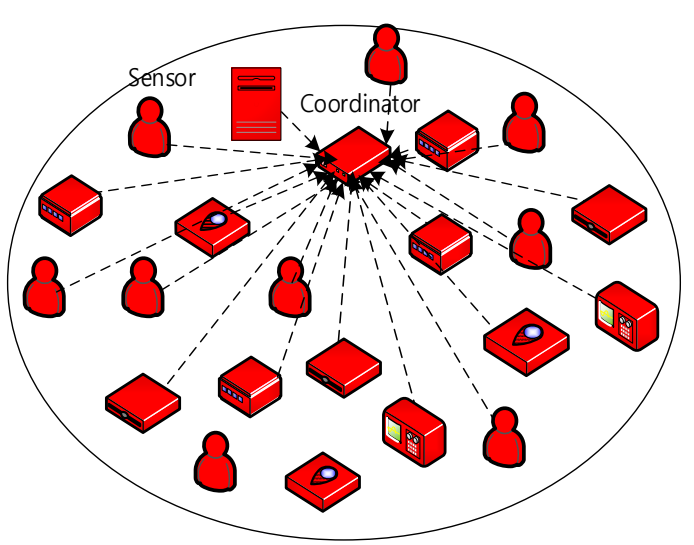

(b)

Figure 2. Traffic congestion model: (a) off/peak time network; (b) peak time network.

Figure 3 shows the total periods of the asynchronous MAC protocol at the peak time and the off/peak time, respectively, as $T_{1}$ and $T_{2}$, where the number of nodes is represented by $D_{1}$ and $D_{2}$.

$P$ represents a period in an asynchronous MAC and always has a constant $P$ regardless of the network traffic congestion. Therefore, the existing asynchronous MAC protocols do not consider the traffic situation, so unnecessary energy consumption occurs in the case of a small number of devices. Moreover, unnecessary energy wastage and data transmission delay occurs due to the inability to receive data even though data reception is required in the opposite case.

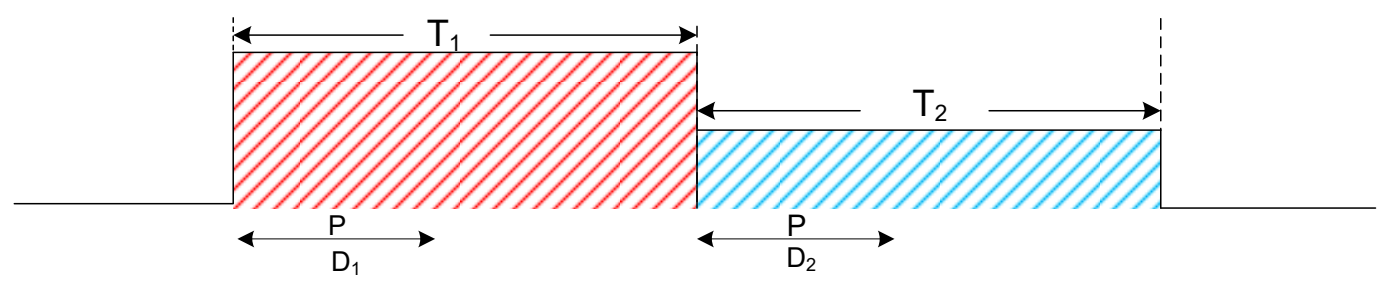

(a) Peak time period

(b) Off/ Peak time period

Figure 3. Amount of data over time: asynchronous period. 


\subsection{The Conventional Scheme}

The period in the existing legacy MAC is always fixed, and $\mathrm{P}$ is set to one period. The transmission energy and the reception energy are expressed by Equations (1) and (2). The parameter settings related to this are shown in Table 1.

$$
\begin{aligned}
& E_{1}=\left(\frac{P}{2} \cdot t_{\text {pre }}\right) \cdot \operatorname{txp}+t_{\text {eack }} \cdot \operatorname{rxp}+t_{\text {data }} \cdot \operatorname{txp}+t_{\text {ack }} \cdot \operatorname{txp} \\
& E_{2}=\left(\frac{P}{2} \cdot t_{\text {pre }}\right) \cdot \operatorname{rxp}+t_{\text {eack }} \cdot \operatorname{txp}+t_{\text {data }} \cdot r x p+t_{\text {ack }} \cdot \operatorname{txp} .
\end{aligned}
$$

The energy consumed to successfully transmit one datum is $E=E_{1}+E_{2}$. In addition, the average delay time required to successfully transmit one datum is expressed by Equation (3).

$$
D=\left(\frac{P}{2} \cdot t_{\text {pre }}+t_{\text {eack }}+t_{\text {data }}+t_{\text {ack }}\right)
$$

Table 1. Symbols for equations: asynchronous MAC protocol.

\begin{tabular}{cl}
\hline Symbol & \multicolumn{1}{c}{ Quantity } \\
\hline$T_{1}, T_{2}$ & Total period of peak time (burst traffic) and off/peak time (normal traffic) \\
$P$ & One period of asynchronous MAC \\
$D_{1}, D_{2}$ & Devices of peak time and off/peak time \\
$D_{P 1}, D_{P 2}$ & Transmission delay of peak time and off/peak time \\
$t_{\text {pre }}$ & Short preamble duration \\
$t_{\text {eack }}$ & Early Acknowledgement duration \\
$t_{\text {ack }}$ & Data Acknowledgement duration \\
$t_{\text {data }}$ & Data duration \\
$\tau$ & Slot time \\
$t x p$ & Transmission power \\
$r x p$ & Reception power \\
\hline
\end{tabular}

In the conventional asynchronous MAC protocol, regardless of the traffic congestion level of the transmitting node, the receiving node always wakes up at period $P$. Therefore, when the transmitting node does not send data, the receiving node wakes up unnecessarily, thus wasting energy. In the opposite case, when many transmitting nodes transmit data, the receiving node wakes up at a constant $P$ period, so the receiver cannot receive the data sent by the transmitting node during the sleep period.

\section{The DDC-MAC Algorithm}

The proposed algorithm increases the duty-cycle ratio of the receiving node at peak time and broadcasts the $\mathrm{CW}$ information through E-ACK to reduce transmission delay and energy consumption compared to the existing transmission and reception duty-cycle ratio protocols.

In WSNs, IoT devices are expected to rapidly change the amount of traffic over time. Experiments were carried out by setting the peak time at which the amount of traffic increases at a specific time and by setting the off/peak time, which is the opposite.

\subsection{The Algorithm and Construction of the Dynamic Duty-Cycle MAC Protocol}

If the peak time starts, it is important for the receiving node to properly adjust the duty-cycle ratio. Figure 4 and Table 2 illustrate the situation; each parameter is used in determining the appropriate receiving node cycle according to the number of DDC-MAC transmitting nodes. 


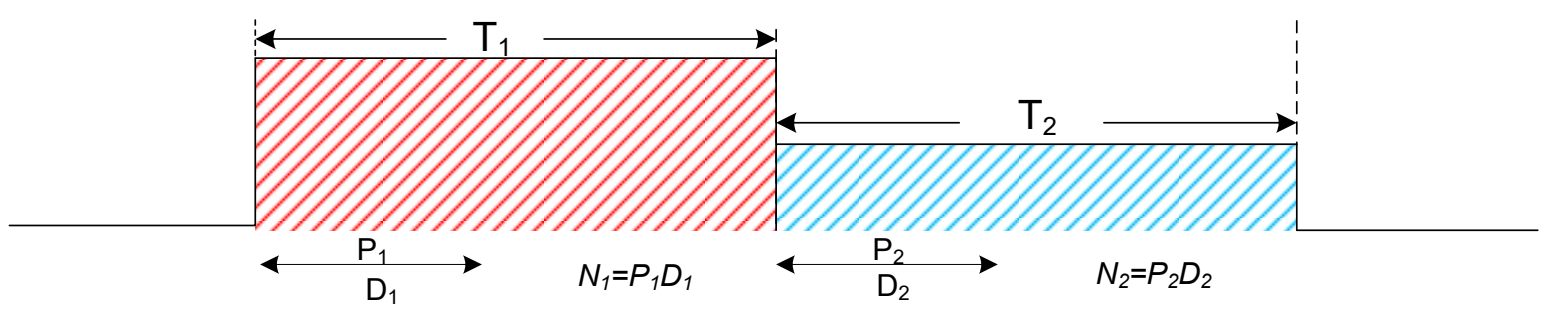

Peak time Network

Off/ Peak time Network

Figure 4. Amount of data over time: proposed asynchronous period.

The period of $T_{1}$ is divided into a peak time period, and the period of $T_{2}$ is divided into an off/peak time period. The $P_{1}$ means one cycle during the peak time period, and $P_{2}$ means one cycle during the off/peak time period. Additionally, $\alpha$ means the slot time of the CW size of the transmitting node. In Figure $4, N_{1}$ expresses the amount of traffic that $D_{1}$ devices transmit during the interval of $P_{1}$, and $N_{2}$ expresses the amount of traffic that $D_{2}$ devices transmit during the $P_{2}$ interval.

Table 2. Symbols for equations: DDC-MAC.

\begin{tabular}{cl}
\hline Symbol & \multicolumn{1}{c}{ Quantity } \\
\hline$T_{1}, T_{2}$ & Total period of peak time (burst traffic) and off/peak time (Normal traffic) \\
$P_{1}, P_{2}$ & New one period of peak time and off/peak time \\
$D_{1}, D_{2}$ & Devices of peak time and off/peak time \\
$D_{P 1}, D_{P 2}$ & Transmission delay of peak time and off/peak time \\
$\alpha$ & Contention window size \\
$N_{1}, N_{2}$ & Amount of network traffic of peak time and off/peak time \\
$t_{\text {pre }}$ & Short preamble duration \\
$t_{\text {eack }}$ & Duration of Early Acknowledgement \\
$t_{\text {ack }}$ & Duration of Data Acknowledgement \\
$t_{\text {data }}$ & Data duration \\
$\tau$ & Slot time \\
$t x p$ & Transmission power \\
rxp & Reception power \\
\hline
\end{tabular}

\subsection{Determining the Period of the Receiving Node Based on the Amount of Traffic}

In order to minimize the transmission delays during the peak time and off/peak time of the receiving node of the proposed DDC-MAC, each new period can be expressed by Equation (4). $X$ defines the assumed simulation network condition.

$$
X=N_{1}\left(\frac{P_{1}}{2}+\alpha \cdot N_{1}^{2}\right) \frac{T_{1}}{P_{1}}+N_{2}\left(\frac{P_{2}}{2}+\alpha \cdot N_{2}^{2}\right) \frac{T_{2}}{P_{2}} .
$$

Equation (4) can be obtained as $P_{1}$ and $P_{2}$, which can minimize the delay due to the variation of $T_{1}$ and $T_{2}$, respectively, assuming there is a constant $\frac{T_{1}}{P_{1}}+\frac{T_{2}}{P_{2}}$. Equations (5)-(8) is the energy consumed to transmit one datum, and Equation (6) is the energy consumed to receive one datum at the receiving node. The total energy can be expressed as $E=E 1+E 2$. Exceptionally, in the $P_{2}$ situation, the energy consumed during $\alpha \cdot \tau$ time for $\mathrm{CW}$ is excluded.

The energy consumption through the new $P_{1}$ and $P_{2}$ determined at peak time and off/peak time is expressed by the following equation. Equations (5) and (6) provide the transmission energy consumed in the periods of $P_{1}$ and $P_{2}$, respectively. Equations (7) and (8) provide the received energy consumed in the periods $P_{1}$ and $P_{2}$.

$$
E 1_{P 1}=\left(\left(\frac{P_{1}}{2} \cdot t_{p r e}+\alpha \cdot \tau\right) \cdot \operatorname{txp}+t_{\text {eack }} \cdot r x p+t_{\text {data }} \cdot \operatorname{txp}+t_{\text {ack }} \cdot \operatorname{txp}\right)
$$




$$
\begin{aligned}
& E 1_{P 2}=\left(\left(\frac{P_{2}}{2} \cdot t_{\text {pre }}\right) \cdot \operatorname{txp}+t_{\text {eack }} \cdot \operatorname{rxp}+t_{\text {data }} \cdot \operatorname{txp}+t_{\text {ack }} \cdot \operatorname{txp}\right) \\
& E 2_{P 1}=\left(\left(\frac{P_{1}}{2} \cdot t_{\text {pre }}\right) \cdot r x p+t_{\text {eack }} \cdot \operatorname{txp}+t_{\text {data }} \cdot \operatorname{rxp}+t_{\text {ack }} \cdot \operatorname{txp}\right) \\
& E 2_{P 2}=\left(\left(\frac{P_{2}}{2} \cdot t_{\text {pre }}\right) \cdot r x p+t_{\text {eack }} \cdot \operatorname{txp}+t_{\text {data }} \cdot \operatorname{rxp}+t_{\text {ack }} \cdot \operatorname{txp}\right) .
\end{aligned}
$$

The average delay time required to successfully transmit one datum is expressed as $P_{1}$ and $P_{2}$, as shown in Equations (9) and (10) below.

$$
\begin{gathered}
D_{P 1}=\left(\frac{P_{1}}{2} \cdot t_{\text {pre }}+\alpha \cdot \tau+t_{\text {eack }}+t_{\text {data }}+t_{\text {ack }}\right) \\
D_{P 2}=\left(\frac{P_{2}}{2} \cdot t_{\text {pre }}+t_{\text {eack }}+t_{\text {data }}+t_{\text {ack }}\right)
\end{gathered}
$$

Figure 5a-c show a flowchart of the dynamic duty-cycle MAC, the frame structure of the E-ACK, and the number of nodes information table (NIT). Figure 5a shows the changed flow chart of seven modules added to the existing asynchronous MAC performed by the receiver. Figure $5 b$ shows the Contention Window (CW) size in the E-ACK frame structure. Figure $5 \mathrm{c}$ is the NIT, which stores the DC and the $\mathrm{CW}$ size information of the receiving node, which changes according to the network traffic.

The whole process in Figure 5a is divided into two operations by peak time and off/peak time in the traffic network. If the receiver has data to receive, Equations (2) and (4) are executed respectively to decrease the sleep period and to increase the $\mathrm{CW}\left(2^{i}\right)$ of the transmitting node by an $i$ increment. In the last sleep period, it is reduced by $\beta$, but the minimum sleep period MIN should be maintained, and the $i$ of the CW size cannot be larger than the maximum M. If Equation (1) is in the opposite situation, Equations (3) and (5) are executed to increase the sleep period and to decrease $i$ by 1 to reduce the CW $\left(2^{i}\right)$ of the transmitting node. It should be increased by $\beta$ in the last sleep period, but should be less than the maximum sleep period MAX, and $i$ should be larger than 0 . After that, the NIT table of Equation (6) is updated with changed information, and the CW size information is loaded in Equation (7) and broadcasted through the E-ACK. Figure $5 b$ shows the addition of the changed $\mathrm{CW}$ size information according to the traffic of the transmitting node in the E-ACK frame structure.

Figure $5 c$ shows the CW size information added through E-ACK as the NIT of the transmitting node, the peak time of traffic, the DC ratio, and the effective time (ET). 


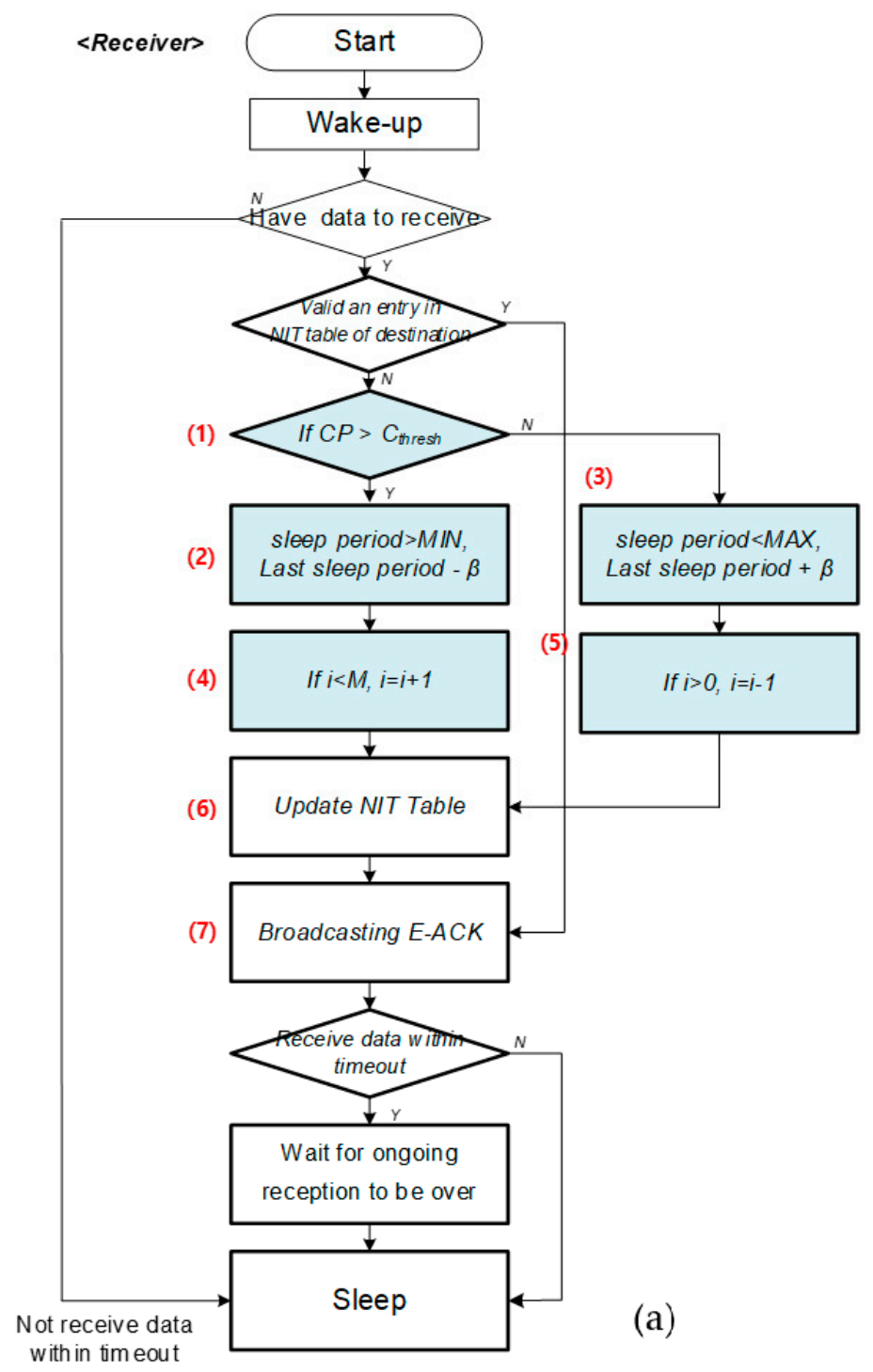

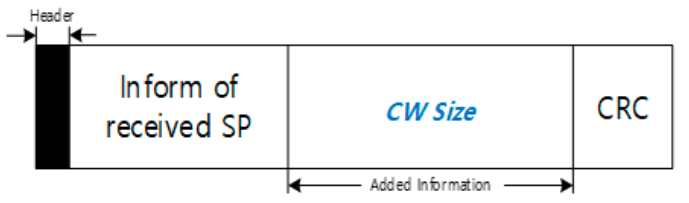

(b)

\begin{tabular}{c|c|c|c}
\hline Peak time & $D C$ & $i$ & $E T$ \\
\hline$\alpha$ hour & $x \%$ & {$[0, \sim M-1]$} & Expirationtime \\
\hline
\end{tabular}

(c)

Figure 5. Structure of the DDC-MAC protocol: (a) algorithm; (b) frame; (c) CW size information.

\section{Experiment Results}

In order to prove the efficiency of the proposed model, we compared the performance of the existing asynchronous duty-cycle MAC protocol and the dynamic duty-cycle MAC (DDC-MAC) protocol under various traffic situations using a mathematical model. It measures the performance of the DDC-MAC algorithm. A new period for minimizing the delay time is presented. The accuracy of the model is verified through the experiment using the ns-2 simulator [21].

Table 3 shows the set values of parameters defined to evaluate the performance of the asynchronous duty-cycle MAC protocol and the DDC-MAC protocol.

We define the power consumption. $R X$ is energy power for receive, $T X$ is energy for transmission, and Idle $x p$ and Sleep $x p$ consume as much power as the receiving data, since a node must listen to the 
media during energy consumption for sleeping. The DC ratio is defined as the ratio of listen time to sleep time. The protocol works on the assumption that the peak time of the network traffic is known in these experiments.

Figures 4-6 indicate the data transmission in the off/peak time and peak time shown in the following experiment by increasing the number of sensor nodes from 10 to 200 .

Table 3. Experiment parameters.

\begin{tabular}{cccc}
\hline Parameter & Value & Parameter & Value \\
\hline Bandwidth & $25 \mathrm{kbps}$ & Duration of E-ACK $(E-A C K)$ & $3 \mathrm{~ms}$ \\
\hline Transmission Range & $100 \mathrm{~m}$ & Data Frame Size $(S)$ & 50 bytes \\
\hline Carrier Sensing Range & $200 \mathrm{~m}$ & Transmission Power $(T X)$ & $52.2 \mathrm{~mW}$ \\
\hline Data Arrival Rate $(\lambda)$ & $1 \mathrm{frame} / \mathrm{s}$ & Reception Power $(R X)$ & $29.1 \mathrm{~mW}$ \\
\hline Duration of Short Preamble $(S P)$ & $3 \mathrm{~ms}$ & Idle Listening Power $($ Idle $x p)$ & $52.2 \mathrm{~mW}$ \\
\hline Contention Window Size $(C W)$ & $2 \sim 8$ slot time $(2 \sim 8 \times 0.5 \mathrm{~ms})$ & Sleeping Power $($ Sleep $x p)$ & $0.001 \mathrm{~mW}$ \\
\hline
\end{tabular}

Figure 6 compares the throughput, latency, and energy consumed by one node at off/peak time and peak time compared to the existing asynchronous MAC and our proposed DDC-MAC. One cycle of the transmitting node is $500 \mathrm{~ms}$, the DC ratio is 3\%. The total number of nodes of the off/peak time is 10 , and the total number of nodes of peak time is 200. The asynchronous MAC protocol's period of the receiving node is $250 \mathrm{~ms}$, which is fixed at $6 \%$. The receiving node of the DDC-MAC protocol has a period of $1000 \mathrm{~ms}$ at the off/peak time, and the DC is $1.5 \%$. However, when the peak time is reached, the DC and CW changes to $15 \%$ and 8 .

Figure 6a shows a similar throughput from the beginning to $2 \mathrm{~s}$ when comparing throughput according to the time. However, the asynchronous MAC continues to decrease throughput due to heavy traffic from peak time after $2 \mathrm{~s}$. Nonetheless, since the DDC-MAC increases the DC of the receiving node, the throughput improves because the receiving node frequently wakes up to process the data sent by the transmitting node.

Figure $6 \mathrm{~b}$ represents an average delay time for the transfer of one datum. In the case of off/peak time, asynchronous MAC and DDC-MAC show similar delay times, but from peak time DDC-MAC shows a relatively low data transmission delay time. The asynchronous MAC is delayed up to $500 \mathrm{ms,}$ which is one cycle of the transmitting node. This is because the probability of data transmission drops greatly as the traffic increases. However, the DDC-MAC wakes up the receiving node 10 times more than the off/peak time, if there is data to be transmitted. This indicates it is possible to transmit data with a high probability of success, which shows a relatively lower delay than the asynchronous MAC.

Figure $6 \mathrm{c}$ draws the energy consumed in processing one datum per second. From the peak time, the asynchronous MAC indicates a higher energy consumption. However, DDC-MAC can transmit data with lower energy than the asynchronous MAC. The asynchronous MAC increases the probability of data collision in high traffic, so energy is consumed to sense energy consumption or channel sensing by retransmissions. However, for DDC-MAC to have a high probability of success in data transmission, unnecessary energy waste is required. 


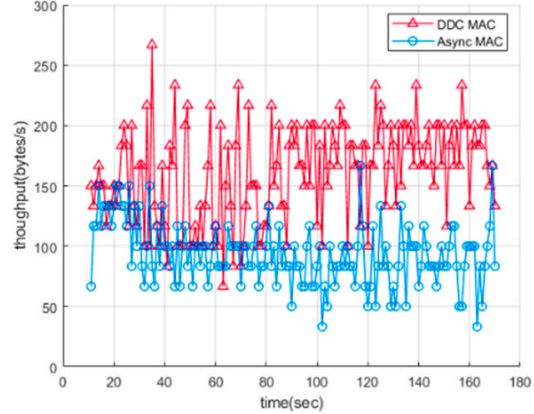

(a)

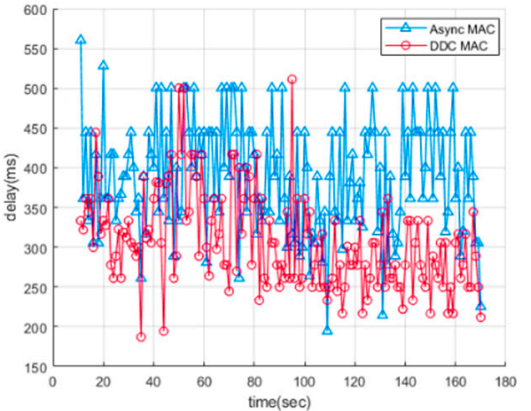

(b)

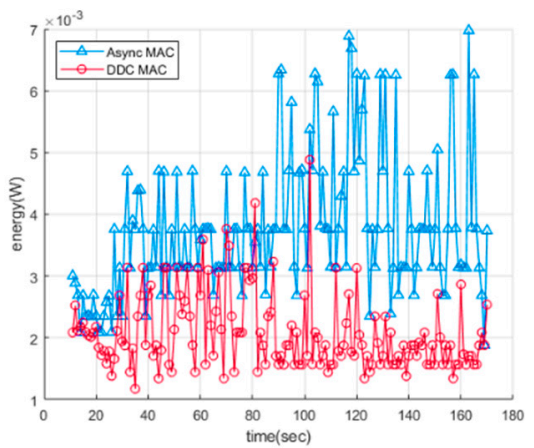

(c)

Figure 6. The comparison of the asynchronous MAC protocol and DDC-MAC traffic amount: (a) throughput; (b) delay; (c) energy consumption/s/node.

Figure 7 represents the throughput, delay, and energy for performance comparison at off/peak time and peak time in DDC-MAC.

Figure 7a shows throughput in peak time and off/peak time, which indicates a higher throughput than the peak time ratio of $10 \%$ at a peak time ratio of $50 \%$. In the case of traffic at $50 \%$, it is necessary to process a large amount of data. This is because the higher the processing efficiency, the higher the possibility of data processing, since the receiving node frequently occurs.

Figure $7 \mathrm{~b}$ shows the delay time for one node to successfully transmit data. As the peak time ratio increases, the frequency of the receiving node for transmitting the generated data increases. Therefore, the data transmission delay is lower at a peak time ratio of $50 \%$ than it is at a peak time ratio of $10 \%$.

Figure $7 \mathrm{c}$ compares the energy consumed by changing the peak time ratio at peak time and off/peak time. At a 10\% peak time ratio, a similar amount of energy is consumed, even though the DDC algorithm is not applied at off/peak time. However, the figure shows that about $60 \%$ of the energy consumption is saved when the DDC algorithm is applied in a traffic environment with a high peak time ratio. 


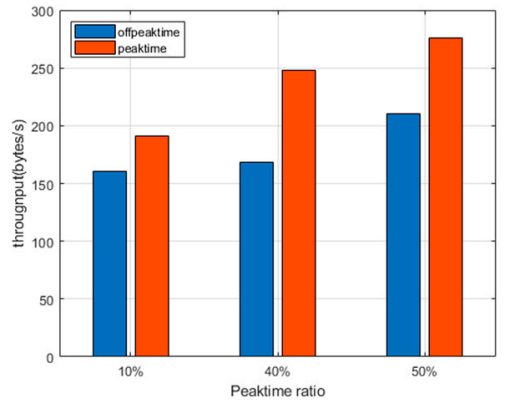

(a)

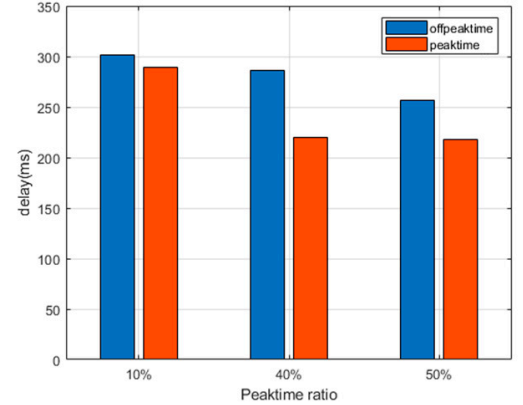

(b)

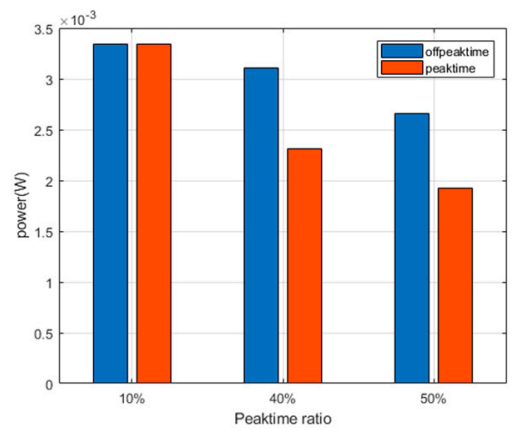

(c)

Figure 7. The comparison of the DDC-MAC performance between off/peak time and peak time: (a) throughput; (b) delay; (c) energy consumption/s/node.

Figure 8 compares the peak time performance of the asynchronous MAC and the DDC-MAC with the peak time ratio.

Figure 8a show the delay time according to the change in the peak time ratio. The asynchronous MAC does not seem to be affected by the change in peak time ratio, but DDC-MAC shows a noticeable relative decrease in latency at $40 \%$, rather than $10 \%$, and does not change from more than $40 \%$. The asynchronous MAC has a $10 \%$ energy consumption at the peak time ratio. Figure $8 \mathrm{~b}$ shows the energy consumption according to the change in peak time ratio.

Due to the high probability at the receiving node, which occurs when data is transmitted, energy consumption is high due to transmission failure. In comparison, a $40 \%$ peak time ratio confirms that the relatively low energy consumption of the receiving node is more likely to receive data due to high data transmission. In the case of $40 \%$, as data transmission increases, the possibility of the receiving node increases and the energy consumption becomes relatively low. However, when the peak time ratio is $50 \%$, the energy consumption increases due to the high collision probability, since the data transmission of the nodes is tested continuously.

In the DDC-MAC, as the peak time of the receiving node increases, the duty-cycle ratio increases, and the CW operates. Therefore, the probability of receiving data successfully without a collision increases. As a result, the data collision probability and retransmission are lowered, and the energy consumption for data transmission is thus lower than that of the asynchronous MAC. However, the change in energy consumption of the DDC-MAC does not greatly improve from $40 \%$ or more. 


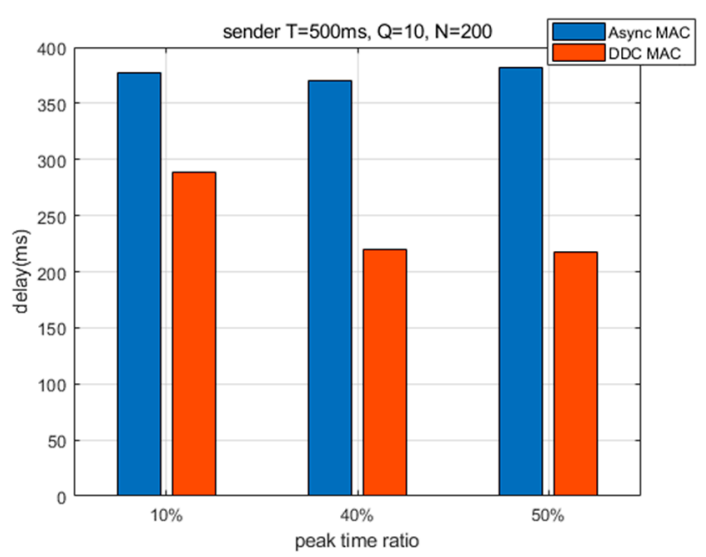

(a)

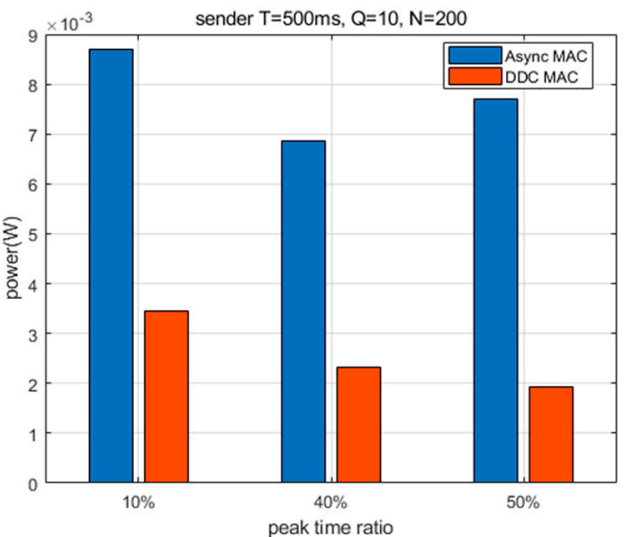

(b)

Figure 8. The comparison of latency and energy consumption of the asynchronous MAC and DDC-MAC in peak time traffic: (a) delay; (b) energy consumption/s/node.

\section{Conclusions}

We propose here a DDC-MAC algorithm that adaptively adjusts the duty-cycle ratio and the CW size of a receiving node according to network congestion. The DDC-MAC algorithm increases the number of wake-ups of the receiving node according to the traffic congestion and changes the $\mathrm{CW}$ size of the transmitting node, thereby reducing collisions in an effort to reduce the delay time and the energy consumption. Thus, the proposed MAC protocol is an improvement in terms of performance in network traffic.

The results of the experiment and mathematical analysis indicate that, when using the DDC-MAC algorithm in heavy traffic environments, the energy required to transmit one datum is reduced by up to about $1 / 4$ and the average service delay is reduced by about $180 \%$.

Author Contributions: Idea and conceptualization: G.K. and J.-G.K.; methodology: G.K., J.-G.K., and M.R.; software: J.-G.K.; analysis: G.K., J.-G.K., and M.R.; visualization: G.K.; development of the joint design scheme and paper writing: G.K.; direction of the research and contribution to the refinement of the algorithm: M.R.; performance discussion: J.-G.K.; paper revision: G.K. and J.-G.K.; project administration: M.R.

Funding: This research was supported by the Basic Science Research Program through the National Research Foundation of Korea (NRF) funded by the Ministry of Education (No. 2018R1D1A1B07049758), the National Research Foundation of Korea (NRF) grant funded by the Korea government (MSIT) (No. 2019R1F1A1056963), and the Samsung Research Funding \& Incubation Center for Future Technology (No. SRFCIT1702-13).

Conflicts of Interest: The authors declare no conflict of interest.

\section{References}

1. Fuhong, L.; Qian, L.; Xianwei, Z.; Yueyun, C.; Daochao, H. Cooperative differential game for model energy-bandwidth efficiency tradeoff in the Internet of Things. China Commun. 2014, 11, 92-102. [CrossRef]

2. Ye, W.; Heidemann, J.; Estrin, D. An Energy-efficient MAC Protocol for Wireless Sensor Networks. In Proceedings of the Twenty-First Annual Joint Conference of the IEEE Computer and Communications Societies, New York, NY, USA, 23-27 June 2002; pp. 1567-1576.

3. Lee, J.; Kim, J.-W. A delay-tolerant virtual tunnel scheme of asynchronous MAC protocol in WSN. Wirel. Pers. Commun. 2013, 70, 657-675. [CrossRef]

4. Khameis, A.; Rashed, S.; Abou-Elnour, A.; Tarique, M. ZigBee Based Optimal Scheduling System for Home Appliances in the United Arab Emirates. Netw. Protoc. Algorithms 2015, 7, 60-80.

5. Park, I.; Yi, J.; Lee, H. A Receiver-Initiated MAC Protocol for Wireless Sensor Networks Based on Tree Topology. Int. J. Distrib. Sens. Netw. 2015, 11, 10. [CrossRef]

6. Othman, M.F.; Shazali, K. Wireless Sensor Network Applications: A Study in Environment Monitoring System. Procedia Eng. 2012, 41, 1204-1210. [CrossRef] 
7. Lloret, J.; Tomas, J.; Canovas, A.; Parra, L. An Integrated IoT Architecture for Smart Metering. IEEE Commun. Mag. 2016, 54, 50-57. [CrossRef]

8. Zhu, Y.; Song, J.; Dong, F. Applications of wireless sensor network in the agriculture environment monitoring. Procedia Eng. 2011, 16, 608-614. [CrossRef]

9. De Craemer, K.; Deconinck, G. Analysis of State-of-the-art Smart Metering Communication Standards. In Proceedings of the 5th Young Researcher's Symposium, Leuven, Belgium, 29-30 March 2010.

10. Soliman, H.; Sudan, K.; Mishra, A. A smart forest-fire early detection sensory system: Another approach of utilizing wireless sensor and neural networks. In Proceedings of the SENSORS, Kona, HI, USA, 1-4 November 2010.

11. Karia, A.; Aseri, T.C. Survey of synchronous MAC protocols for Wireless Sensor Network. In Proceedings of the Recent Advances in Engineering and Computational Sciences (RAECS), Chandigarh, India, 6-8 March 2014.

12. Huang, P.; Xiao, L.; Soltani, S.; Mutka, M.W.; Xi, N. The evolution of MAC protocols in wireless sensor networks: A survey. IEEE Commun. Surv. Tutor. 2012, 15, 101-120. [CrossRef]

13. Van Dam, T.; Langendoen, K. An Adaptive Energy-Efficient MAC Protocol for Wireless Sensor Networks. In Proceedings of the 1st International Conference on Embedded Networked Sensor Systems, Los Angeles, CA, USA, 5-7 November 2003; pp. 171-180.

14. Kumar, S.S.; Kumar, M.N.; Sheeba, V.S.; Kashwan, K.R. Power Efficient Dynamic MAC Protocol (D-MAC) for Wireless Sensor Networks. J. Inf. Comput. Sci. 2012, 9, 1795-1805.

15. Liu, Y.; Jiang, F.; Liu, H.; Wu, J. SC-MAC: A sender-centric asynchronous MAC protocol for burst traffic in wireless sensor networks. In Proceedings of the 18th Asia-Pacific Conference on Communications, Jeju Island, Korea, 15-17 October 2012; pp. 848-853.

16. Polastre, J.; Hill, J.; Culler, D. Versatile Low Power Media Access for Wireless Sensor Networks. In Proceedings of the 2nd International Conference on Embedded Networked Sensor Systems, Baltimore, MD, USA, 3-5 November 2004; pp. 95-107.

17. Yang, O.; Heinzelman, W. Modeling and performance analysis for duty-cycled MAC protocols with applications to S-MAC and X-MAC. IEEE Trans. Mob. Comput. 2012, 11, 905-921. [CrossRef]

18. Enz, C.C.; El-Hoiyadi, A.; Decotignie, J.D.; Peiris, V. WiseNet: An Ultralow power wireless sensor network solution. Computer 2004, 37, 62-70. [CrossRef]

19. Sabti, H.A.; Thiel, D.V. A study of Wireless Communication Links on a Body Centric Network During Running. Procedia Eng. 2014, 72, 3-8. [CrossRef]

20. Negra, R.; Jemili, I.; Belghith, A. Wireless Body Area Networks: Applications and Technologies. Procedia Eng. 2016, 83, 1274-1281. [CrossRef]

21. NS-2 Simulator Official Site. Available online: http://www.isi.edu/nsnam/ns (accessed on 5 August 2019). 